\title{
Spatial probability learning by cats '
}

STEPHEN F, POLAND AND J. M. WARREN

THE PENNSYLVANIA STATE UNIVERSITY

Groups of six cats were trained, with noncorrection or with guidance, for 1200 trials on each of four ratios of reinforcement: 70:30, 60:40, 40:60 and 30:70, in a spatial probability learning experiment. Both groups eventually learned to choose the more frequently rewarded stimulus on almost every trial, the noncorrection Ss learning to do so much sooner than the guidance Ss. The results of this experiment with cats tend to agree with previous observations that rats and monkeys maximize on spatial probability problems. The available evidence, however, indicates that cats tested with guidance on spatial probability problems learn to maximize considerably more slowly than rhesus monkeys and that monkeys are, in turn, inferior to rats.

Bitterman (1965a, b) maintains that vertebrate classes differ qualitatively in performance on probability learning tasks. This view implies that there should be no major qualitative differences among species of the same class. The available data are, however, too limited to provide a realistic estimate of intraclass variability; the mammals, for example, are represented only by rats and monkeys. The purpose of the present experiment was to determine whether the choice behavior of cats on a spatial probability problem is qualitatively similar to that of rats and monkeys tested under analogous conditions. This investigation was motivated by the observation of rather impressive differences between the performance of cats and monkeys in visual probability learning (Warren \& Beck, 1966).

\section{Method}

Twelve mature cats, nine mongrel and three Siamese, were studied. All of the Ss had previously served in learning set experiments, and all had been trained on visual probability problems, but none had participated in any experiment on spatial probability learning before this experiment.

The cats were tested in the WGTA. The manipulanda were identical $2.5 \times 4.0$ rectangles cut from $3 / 4$ in. lumber which were painted dark gray, and presented on a medium gray test tray, containing two food wells 12 in. apart.

The Ss were tested 1200 trials on each of four ratios of reinforcement in the following sequence: $70: 30,60: 40,40: 60$, and $30: 70$, with 50 trials being given in each test session and five sessions per week. The 12 Ss were assigned to two groups of six animals each, the Noncorrection and Guidance groups. The Noncorrection group was trained with a strict noncorrection procedure throughout the experiment; $S$ was allowed only a single response on each trial. For Guidance Ss each trial ended with a reinforced response. When the cat's initial response on a given trial was incorrect, the opaque screen of the WGTA was lowered, the object on the nonrewarded side of the tray was positioned behind the empty food well, and the baited food well remained covered with its object. The test tray was then presented to $S$, and the trial ended only when it displaced the object covering the rewarded food well and secured the reinforcement. Only initial responses were considered in analyzing the results.

For half the Ss in each group, the initially preferred position was the more frequently reinforced (major) stimulus, and for half, the initially preferred position was the less frequently reinforced (minor) stimulus on the $70: 30$ problem.

The standard reinforcement was a $1 / 4$ in. cube of pork kidney, a highly preferred food; the Ss were tested under approximately $23 \mathrm{~h}$ of food deprivation. Results

The results are summarized in Fig. 1, a plot of median percent choices of the original major stimulus by the Noncorrection and Guidance groups, as a function of successive blocks of 100 trials. Solid lines represent the level which would be predicted by a matching model on each ratio, and dashed lines indicate the level which lies just half way between those that would correspond to matching and to perfect maximizing.

It is readily apparent that the Noncorrection group quickly learned to maximize on the $70: 30$ ratio and continued to do so throughout testing on the $60: 40$ ratio of reinforcement. The Guidance group performed at a higher than matching level on 70:30 and 60:40 ratios, but, on both ratios, approximated matching levels more closely than maximizing levels of reinforcement. U-tests indicate that on both these

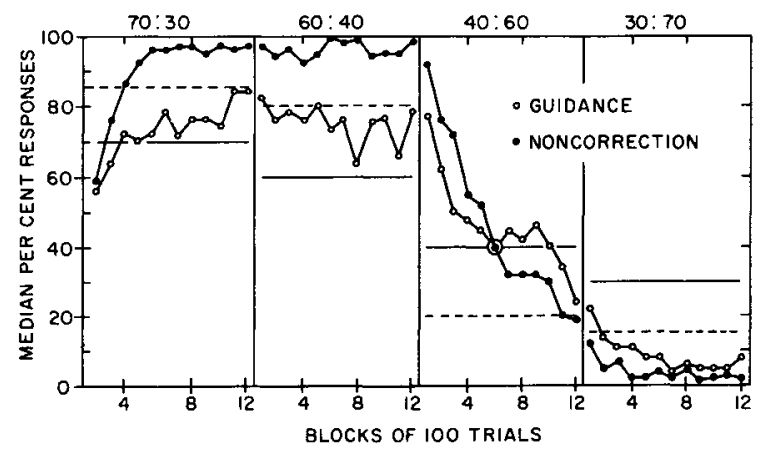

Fig. 1. Median percentage of responses to the initial major stimulus. 
ratios the number of choices of the major stimulus by the Guidance group was significantly less than for the Noncorrection group.

The Noncorrection group learned the reversal from $60: 40$ to $40: 60$ more rapidly than the Guidance group. The groups do not, however, differ significantly in percentage of responses to the major stimulus over the last 100 trials on this ratio. Both the Guidance and Noncorrection cats achieved satisfactory maximizing behavior on the final 30:70 problem.

\section{Discussion}

The results of this investigation indicate that cats, when tested on spatial probability with the noncorrection or guidance methods, learn to select the more frequently reinforced stimulus on almost every trial, and that Ss trained with noncorrection learn to maximize much more quickly than Ss trained with guidance. These findings on cats are generally consonant with the data for rats and monkeys. Rats (Bitterman, Wodinsky, \& Candland, 1958; Roberts, 1966) and monkeys (Wilson, 1960; Wilson \& Rollin, 1959) usually maximize on spatial probability learning tasks when tested with guidance or correction. In addition, it has been shown that monkeys learn to maximize in many fewer trials when tested with noncorrection (Wilson \& Rollin, 1959) than with guidance or correction (Wilson, 1960).

It should, however, be pointed out that there is a wide range of variation among the mammalian species in the rate that they learn to maximize on spatial problems when tested with guidance. The present sample of cats required 3700 trials. Rats apparently maximize on 70:30 spatial problems in about 100 trials (Bitterman, Wodinsky, \& Candland, 1958; Roberts, 1966). Rhesus monkeys (Wilson, 1960), trained on a 75:25 spatial problem with guidance, attained a level of 90 percent responses to the major stimulus only after 384 trials. The range, and somewhat paradoxical distribution, of species differences in performance on spatial probability tasks suggests the possibility that qualitatively similar behavior, i.e., maximizing, may not reflect completely homologous processes in different mammalian species.

\section{References}

Bitterman, M. E. The evolution of intelligence. Scient. American, $1965 \mathrm{a}, 212,92-100$.

Bitterman, M. E. Phy?etic differences in learning. Amer. Psychologist, $1965 \mathrm{~b}, 20,396-410$.

Bitterman, M. E., Wodinsky, J., \& Candland, D. K. Some comparative psychology. Amer. J. Psychol., 1958, 71, 94-110.

Roberts, W. A. Learning and motivation in the immature rat. Amer. J. Psychol., 1966, 79, 2-23.

Warren, J. M., \& Beck, C. H. Visual probability learning by cats. J. comp. physiol. Psychol., 1966, 61, 316-318.

Wilson, W. A., Jr., Two-choice behavior of monkeys. J, exp. Psychol., 1960, 59, 207-208.

Wilson, W. A. Jr., \& Rollin, A. R. Two-choice behavior of thesus monkeys in a noncontingent situation. J. exp. Psychol., 1959, $58,174-180$.

\section{Note}

1. This research was supported by Grant MH-4726 from the National Institute of Mental Health, United States Public Health Service.

\section{Reply to Goldstein by Ronald Baenninger, Temple University}

Goldstein (1967) has said that I attribute the extinction of visually reinforced responses in Siamese fighting fish to habituation. My argument, however, was more subtle, and can be clarified in the following way.

Habituation is defined by Goldstein (1967) as the waning of a response as ". . a result of repeated presentation of a constant stimulus." The problem is that Goldstein is not clear about which constant stimulus he is talking about. In the visual reinforcement paradigm there are two constant stimuli during acquisition; a ring through which $S$ is supposed to swim, and a mirror which elicits aggressive displaying by $S_{2}$ Extinction refers to a waning of swimming through the ring when the mirror is no longer presented; habituation refers to a waning of aggressive displays to the mirror.

Hinde (1966) reports finding habituation of the aggressive display. My results (Baenninger, 1966) demonstrated that the tendency to approach displayeliciting stimuli may also wane.

I propose that these two types of waning are not independent. If waning of the display response to the mirror occurs, then it seems quite likely that the response of swimming through the ring in extinction trials may also wane more rapidly than if the mirror response had not habituated to some extent. I believe that the situation in Goldstein's experiment is analogous to extinguishing a satiated animal, or at least one who is partially satiated. A number of experiments (Yamaguchi, 1951; Koch \& Daniel, 1945) have demonstrated that rats which are satiated, or partially satiated, just prior to extinction trials do show minimal resistance to extinction when compared to Ss who learned under similar conditions but are deprived in extinction. The unusual acquisition curve, and the precipitous extinction rate found by Goldstein (1967) in his experimental group, may even be explained in this fashion.

\section{References}

Baenninger, R. Waning of aggressive motivation in Betta splendens. Psychon. Sci.. 1966, 4, 241-242.

Goldstein, S. R. Mirror image as a reinforcer in Siamese fighting fish: a repetition with additiunal controls. Psychon. Sci., 1967, $7,331-332$.

Hinde, R. A. Animal Behaviour. New York: McGraw-Hill, 1966

Koch, S., \& Daniel, W. J. The effect of satiation on the behavior mediated by a habit of maximum strength. J. exp. Psychol., 1945, $35,167-187$.

Yamaguchi, H. G. Drive (D) as a function of hours of hunger (h). J. exp. Psychol., 1951, 42, 108-117. 